\title{
Tests of the APS X-ray Transmitting Beam Position Monitors at ESRF
}

Deming Shu, Juan Barraza, Tuncer M. Kuzay, Advanced Photon Source, Argonne National Laboratory, 9700 S. Cass Av. Argonne, IL 60439, U.S.A. and

Graham Naylor, Pascal Elleaume, European Synchrotron Radiation Facility, BP 220, F-38043

RECFIVED

Grenoble, Cedek, France

Abstract JUI 31997

Two differetyos of pynthetic diamond-based Xray transmitting beam position monitor (XBPM) prototypes have been studied with an undulator white beam at the European Synchrotron Radiation Facility (ESRF) ID-6 beamline. Of particular interest was the possibility of designing an integral window and filter/photon beam position monitor for the Advanced Photon Source (APS) high heat flux insertion device beamlines.

The photoelectron-emission type transmitting XBPM prototype uses a 25 -mm-diameter, 175-micron-thick CVDdiamond disk with 0.2-micron-thick electrically isolated patterns of aluminum coating on one side of the diamond disk. The photoelectron emission signal was collected from the aluminum-coat surface to provide the beam position information.

A novel photoconductive-type transmitting XBPM prototype uses the same CVD-diamond disk, but patterns of aluminum coating were applied on both sides of the diamond disk. A DC bias voltage was used to generate the current signal, which is based on photoconductive properties of the CVD-diamond. Test results are presented in the paper.

\section{INTRODUCTION}

There are many challenging tasks in the design of insertion device (ID) beamline instrumentation that relate to high heat load and high heat flux problems. One such component is the X-ray beam position monitor (XBPM) for the ID beamlines.

Synthetic diamond has been proposed for use as an XBPM blade material for the ID front end of the APS project [1]. Synthetic diamond, such as CVD diamond, offers superior thermal-physical properties, such as high thermal conductivity, low thermal expansion coefficient, and good mechanical strength and stiffness under heat. In the classic photoelectron emission-type XBPM, the monitor blade is mounted parallel to the radiation beam direction to eliminate the total thermal power absorbed by the blade. To reduce the heat power density on the blade edge surface, inclined edge geometry is usually utilized.
A photoelectron emission-type X-ray transmitting beam position monitor (TBPM) has been proposed for combining filter/window and XBPM functions [2]. The basic concept of the TBPM is to mount the monitor blade perpendicular to the synchrotron radiation beam and to design the blade and its sensor coating in such a way that most of the $\mathrm{x}$-ray beam will be transmitted through the blade. Thus, the total absorbed photon power cannot cause thermal damage to the blade. Earlier a sample monitor was tested at NSLS on the X-25 wiggler beamline for proof of the principle [3]. Based on the success of this preliminary test with a focused wiggler beam, a compact filter/mask/window assembly was designed for undulator beamline commissioning activity at the APS beamlines [4].

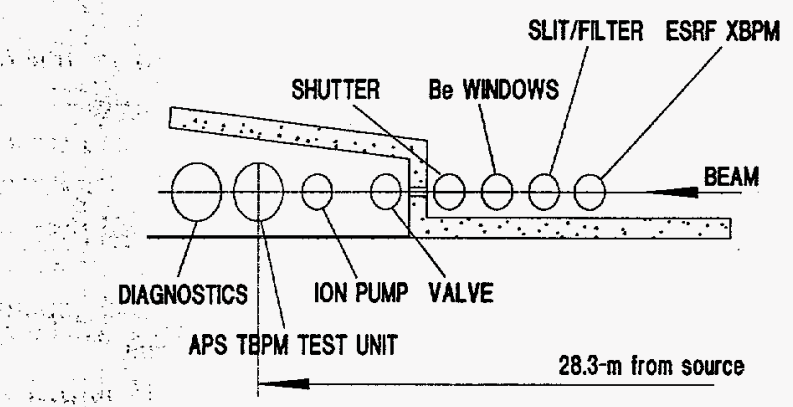

Fig. 1. Schematic layout of the ESRF ID-6 beamline.

Similar to the photoelectron emission-type TBPM, a novel photoconductive-type TBPM has also been proposed [5]. To test this design with a full power undulator beam, a prototype TBPM test base has been built. Two different types of synthetic-diamond-based TBPM test samples were prepared at the APS. The test plan at the ESRF beamline ID-6 was part of the Tripartite Joint Research Agreement Protocol among the APS, ESRF and SPring-8 synchrotron radiation facilities.

\section{BEAMLINE TEST SETUP}

The ID- 6 beamline at the ESRF has a 46-mm-period, 1.66-meter-long undulator source. During the time we were doing our experiments, the ESRF storage ring was running in hybrid mode with a maximum of $140 \mathrm{~mA}$ current. The 


\section{DISCLAIMER}

This report was prepared as an account of work sponsored by an agency of the United States Government. Neither the United States Government nor any agency thereof, nor any of their employees, makes any warranty, express or implied, or assumes any legal liability or responsibility for the accuracy, completeness, or usefulness of any information, apparatus, product, or process disclosed, or represents that its use would not infringe privately owned rights. Reference herein to any specific commercial product, process, or service by trade name, trademark, manufacturer, or otherwise does not necessarily constitute or imply its endorsement, recommendation, or favoring by the United States Government or any agency thereof. The views and opinions of authors expressed herein do not necessarily state or reflect those of the United States Government or any agency thereof. 


\section{DLCCAMMER}

Portions of this doecoment mey be illegible in electronic image prodnets. Images are produced from the best arainable original docoment 
undulator was operating at a gap in the $21 \mathrm{~mm}$ to $41 \mathrm{~mm}$ range.

Fig. 1 shows a schematic of the ID-6 layout. During the test, an APS TBPM test unit was installed in the first optics enclosure (FOE). Upstream of the APS TBPM testunit, a 500-micron-thick pyrolitic graphite filter and two 250-micron-thick Beryllium windows cut off most of the soft X-ray from the source. Downstream of the test unit, a fluorescent screen with a graduated aluminum filter and a CCD camera provides the capability to monitoring the beam profile and helps in the system alignment.

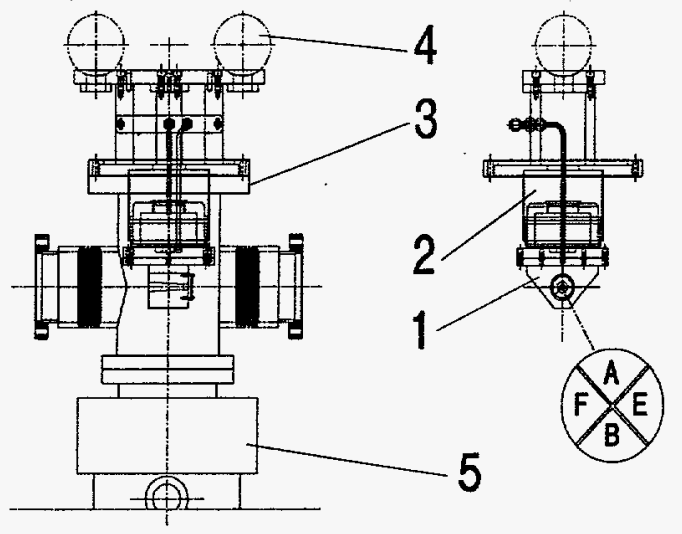

Fig. 2. The APS TBPM test-unit (1)sample mounting block ; (2) water-cooling base; (3) vacuum chamber; (4) survey-mark; (5) stepping-motor-driven stage.

The TBPM test unit was designed to be compatible with the ESRF XBPM vacuum chamber and its supporting stages. As shown in Fig. 2, the APS TBPM test unit consists of six components. The TBPM sample is assembled on a sample mounting block (1), which is cooled by a water-cooling base (2). The water-cooling base is mounted on the top of the ESRF XBPM vacuum

chamber (3). There are two survey marks (4) on the mounting flange for alignment purpose. The vacuum chamber is supported by a set of stepping-motor-driven stages (5), which provide precise horizontal and vertical motions for fine alignment and scan motion for the TBPM test. An optical encoder with 0.1 micron resolution was added to the stage to verify the motion accuracy.

\section{TESTS OF THE PHOTOEMISSION-TYPE XBPM}

The test sample for photoelectron emission-type TBPM uses a 25-mm-diameter, 175-micron-thick CVDdiamond disk with 0.2 -micron-thick electrically isolated quadrant patterns of an aluminum coating on one side of the diamond disk. Fig. 4 shows the CVD-diamond disk and its clamping and cooling structure. The photoelectron emission signal was collected from the aluminum-coated surface to provide the beam position information.
Three different sample setups were arranged for the experiments as shown in Fig. 3. In configuration (a), we used same clamping and cooling structure as we did in the NSLS X-25 test. The aluminum-sensor coating was facing the X-ray beam, and an alumina insulator was applied to isolate the sensor from the cooling base electrically but retain reasonable thermal conduction for cooling.

Configuration (b) was designed to test whether we can use the CVD-diamond as an electric insulator for the aluminum sensor coating to improve the disk cooling efficiency. So that, in configuration (b), the aluminum coating on the CVD-diamond disk was mounted facing downstream to the beam direction and no insulator applied between the copper cooling base and the CVD-diamond disk.

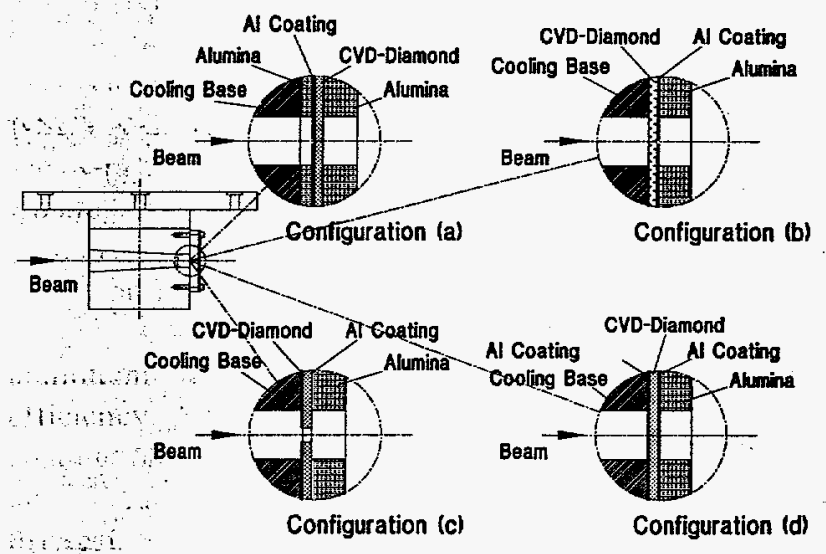

Fig. 3. Sample set-up configurations for the TBPM test.

The test configuration (c) was similar to (a), except a $1.5 \mathrm{~mm}$ through hole was made in the center of the CVDdiamond disk to allow a clear X-ray beam path.

The test results show that all of these three configurations provide good beam position sensitivity. Fig. 4 shows the output signal from the TBPM test sample in configuration (c) as a function of the stage vertical motion.

\section{TESTS OF THE PHOTOCONDUCTIVE-TYPE XBPM}

Since 1975, many researchers have explored the photoconductive properties of natural diamond with X-rays and gamma rays [6]. The photoconductive properties of CVD diamond have also been exploited [7]. A test sample for a novel photoconductive-type TBPM was designed and prepared at the APS as shown in Fig. 3 configuration (d). The sample was made from the same type of CVDdiamond disk as was used in the photoemission-type TBPM. But, the quadrant patterns of the aluminum coating were applied on both sides of the diamond disk. A DC bias voltage was used to generate the current signal, which is based on photoconductive properties of the CVD- diamond. 
Fig. 5 shows the output signal from the photoconductive TBPM test sample in configuration (d) as a function of the stage vertical motion. During this test, the bias voltage applied to the CVD-diamond disk was $1.5-$ volts.

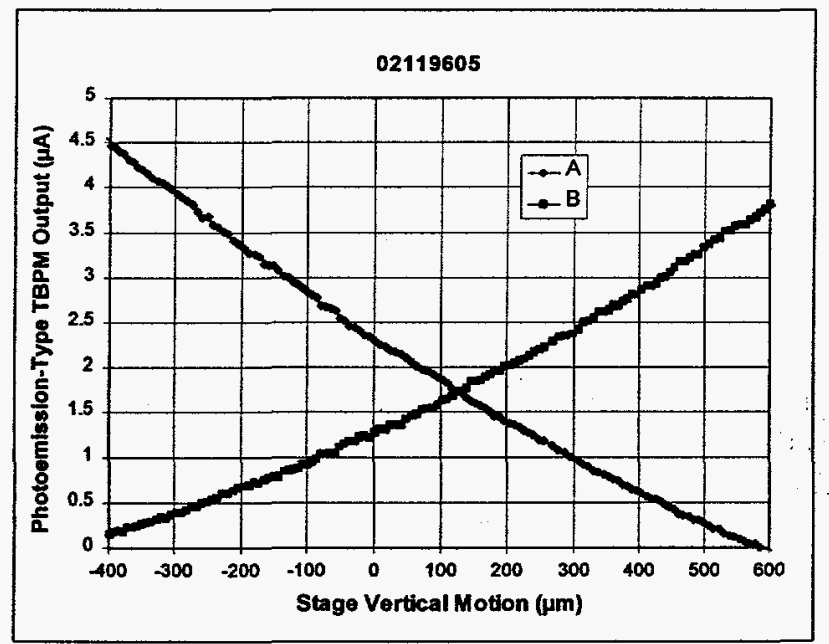

Fig. 4. Output signal from a photoemission-type TBPM test sample in configuration (c) as a function of the stage vertical motion. ID-6 undulator gap was $28.2-\mathrm{mm}$. ESRF storage ring current was $121-\mathrm{mA}$

\section{DISCUSSION}

According to the experiences obtained with the XBPMs in many synchrotron radiation laboratories, contamination signals caused by the bending-magnet (BM) emitted radiation become a major problem. Problems are exacerbated for the XBPM when the insertion devices (IDs) operate with different magnet gaps, because the percentage level of the contamination will be a variable.

From the test results, we have learned that, compared to a photoemmision-type TBPM, the beam position signal from a photoconductive-type TBPM has less ID gap dependence. This maybe caused by the higher sensitivity of the photoconductive type TBPM to the hard X-ray radiation.

\section{ACKNOWLEDGEMENTS}

This work was supported by the U.S. Department of Energy, BES-Materials Science, under contract W-31-109Eng-38.

\section{REFERENCES}

[1]. D. Shu, B. Rodricks, T. Sanchez, and T.M. Kuzay, "The APS X-ray undulator photon beam position monitor and tests at CHESS and NSLS", Nucl. Instru. and Meth. A319(1992)56-62.

[2]. U.S. patent 5,404,014 (Feb. 1994)

[3]. D. Shu, J.T. Collins, J. Barraza, and T.M. Kuzay, "The APS X-ray transmitting beam-position-monitor tests at the NSLS X-25 beamline", Nucl. Instru. and Meth. A347(1994)577-580.

[4]. D. Shu, and T.M. Kuzay, "Design of the commissioning filter/mask/window assembly for undulator beamline front ends at the Advanced Photon Source", Rev. Sci. Instrum. 67(9), Sept. 1996.

[5]. D. Shu, T.M. Kuzay, Y. Fang, J. Barraza, and T. Cundiff, "Synthetic diamond-based position-sensitive photoconductive detector development for the APS", to be submitted for the $6^{\text {th }}$ International Conference on Synchrotron Radiation Instrumentation, Japan, Aug. 1997.

[6]. D.R. Kania, L.S. Pan, P. Bell, O.L. Landen, H. Kornblum, P. Pianetta, and M. D. Perry, "Absolute xray power measurements with subnanosecond time resolution using type lia diamond photoconductors", J. Appl. Phys. 68(1), July 1990.

[7]. R.J Keddy, T.L. Nam, and R.C. Burns, "Synthetic diamonds as ionisation chamber radiation detectors in biological environments", Phys. Med. Biol., Vol.32(6)751-759, 1987.

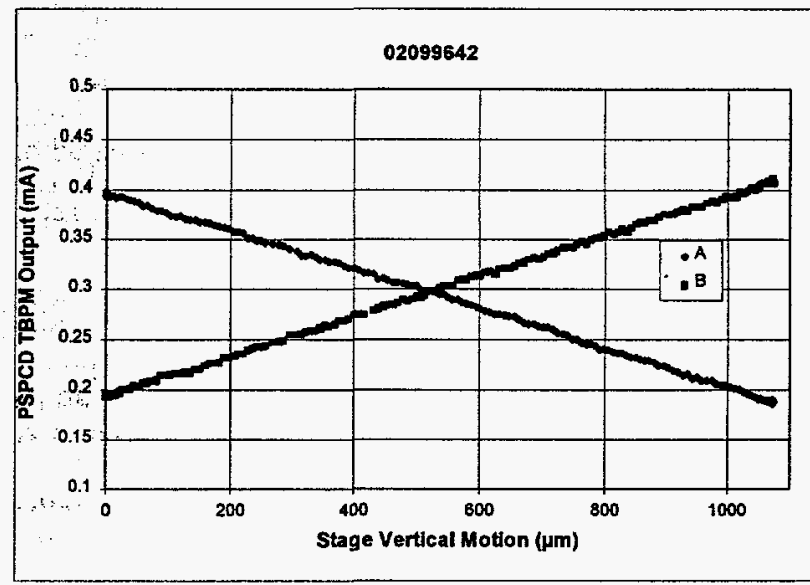

Fig. 5. Output signal from a photoconductive-type TBPM test sample in configuration (d) as a function of the stage vertical motion. ID-6 undulator gap was $29-\mathrm{mm}$. ESRF storage ring current was $110-\mathrm{mA}$. 